\title{
1 Levonorgestrel-releasing intrauterine system use is associated with a \\ 2 decreased risk of ovarian and endometrial cancer, without increased \\ 3 risk of breast cancer. Results from the NOWAC Study \\ 4 \\ Mie Jareid ${ }^{1 *}$, Jean-Christophe Thalabard ${ }^{2}$, Morten Aarflot ${ }^{1}$, Hege M. Bøvelstad ${ }^{1}$, Eiliv Lund ${ }^{1}$, Tonje Braaten $^{1}$
}

1) Department of Community Medicine, Faculty of Health Sciences, UiT - The Arctic University of Norway, Troms $\varnothing$, Norway

2) Applied Mathematics Lab, UMR CNRS 8145, Paris Descartes University, USPC

E-mail addresses:

MJ:mie.jareid@uit.no

JCT: Jean-Christophe.Thalabard@parisdescartes.fr

MAA: morten.arflot@uit.no

HMB: hege.m.bovelstad@uit.no

EL: eiliv.lund@uit.no

TB: tonje.braaten@uit.no

*Corresponding author: Mie Jareid, Department of Community Medicine, Faculty of Health Sciences, UiT The Arctic University of Norway, Pb 6070 Langnes, NO-9037 Troms $\varnothing$, Norway; Telephone: +47 77625209; E-mail: mie.jareid@uit.no

Disclaimer: Some of the data in this article are from the Cancer Registry of Norway. The Cancer Registry of Norway is not responsible for the analysis or interpretation of the data presented.

Word count: 4044, Abstract: 250

Keywords: Epidemiology; prospective cohort study; ovarian neoplasms; uterine neoplasms; breast neoplasms; intrauterine device; intrauterine device, medicated; levonorgestrel; levonorgestrel-releasing intrauterine system; hormonal contraceptives

Abbreviations used: $\mathrm{BMI}$, body mass index; $\mathrm{Cl}$, confidence interval; ICD, International Classification of Diseases; LNG-IUS, levonorgestrel-releasing intrauterine system; NOWAC, Norwegian Women and Cancer; OC, oral contraceptives; PY, person-years; RR, relative risk; SD, standard deviation; SIR, standardized incidence ratio 


\section{ABSTRACT}

33 Objective Women with ovarian cancer have poor survival rates, which have proven difficult to improve;

34 therefore primary prevention is important. The levonorgestrel-releasing intrauterine system (LNG-IUS)

35 prevents endometrial cancer, and recent studies suggested that it may also prevent ovarian cancer, but

36 with a concurrent increased risk of breast cancer. We compared adjusted risks of ovarian, endometrial,

37 and breast cancer in ever users and never users of LNG-IUS.

38 Methods Our study cohort consisted of 104318 women from the Norwegian Women and Cancer Study,

399144 of whom were ever users and 95174 of whom were never users of LNG-IUS. Exposure information

40 was taken from self-administered questionnaires, and cancer cases were identified through linkage to

41 the Cancer Registry of Norway. Relative risks (RRs) with 95\% confidence intervals (Cls) were estimated

42 with Poisson regression using robust error estimates(1).

43 Results Median age at inclusion was 52 years and mean follow-up time was 12.5 (standard deviation

44 3.7) years, for a total of 1305435 person-years. Among ever users of LNG-IUS there were 18 cases of

45 epithelial ovarian cancer, 15 cases of endometrial cancer, and 297 cases of breast cancer. When ever

46 users were compared to never users of LNG-IUS, the multivariable RR of ovarian, endometrial, and

47 breast cancer was $0.53(95 \% \mathrm{Cl}: 0.32,0.88), 0.22(0.13,0.40)$, and $1.03(0.91,1.17)$, respectively.

48 Conclusion In this population-based prospective cohort study, ever users of LNG-IUS had a strongly

49 reduced risk of ovarian and endometrial cancer compared to never users, with no increased risk of

50 breast cancer. 


\section{INTRODUCTION}

54 In 2012, ovarian cancer caused an estimated 152000 deaths worldwide (2). The cumulative risk of

55 ovarian cancer until age 75 is $1.3 \%$ in Norway and is similar in the United States $(3,4)$. The symptoms of ovarian cancer are vague, and there is no screening test. This has led to problems of late diagnosis and a

575 -year survival of less than 50\% (5). Thus, ovarian cancer ranks eighth in cancer incidence, but fifth in

58 cancer mortality among women (4). Primary prevention therefore remains the best available measure

59 against ovarian cancer (5).

Risk of ovarian cancer is reduced by $15-29 \%$ for every 5 years of oral contraceptive (OC) use, and globally, OC use prevents an estimated 30000 cases of ovarian cancer each year (6). Long-term OC use

62 also reduces the risk of endometrial cancer, with 5-9 years of use reducing the risk by $34 \%$ (7). However,

63 OC use increases the risk of breast cancer up to $38 \%$ with more than 10 years use, and for minimum 5

64 years after cessation $(8,9)$ in addition to carrying other health risks. Prescribing OCs for ovarian cancer 65 prevention to women who do not need contraception is not recommended (10).

The levonorgestrel-releasing intrauterine system (LNG-IUS) was introduced in Norway in 1994. In

67 the Nordic countries, LNG-IUS is the second-most used form of contraception after OCs, and it is the most commonly used form of long-acting reversible contraception (11). Recently, three Finnish studies have shown that, compared to the general population, LNG-IUS users have a standardized incidence ratio (SIR) of 0.59 for ovarian cancer and 0.46 for endometrial cancer $(12,13)$, but also an increased risk

71 of ductal and lobular breast cancer (SIR 1.20 and 1.33 respectively, increasing to SIR 1.37 and 1.73 with

72 more than 5 years of use) (14). However, these studies did not adjust for other hormonal risk factors.

Our study aim was to combine self-reported information on OC use and reproductive factors from

74 the Norwegian Women and Cancer (NOWAC) Study, with registry-based follow-up of cancer cases to 75 compare adjusted risks of ovarian, endometrial, and breast cancer in ever users and never users of LNG- 
IUS. We also included estimates of the reduction in the risk of endometrial cancer in this nationally

77 representative cohort, given the well-known preventive effect of LNG-IUS use on this cancer (15).

\section{METHODS}

79 Study cohort

80 The NOWAC Study is a population-based prospective cohort study designed to investigate the

81 association between hormone use and hormone-dependent female cancers (16). During 1991-2007,

82 women born between 1927 and 1965 were randomly selected from the Norwegian Population Registry

83 and were sent a questionnaire along with a letter that explained the study. Those who returned a

84 completed questionnaire were enrolled. Statistics Norway replaced participants' names and personal identification numbers with serial numbers for use by researchers. Recruitment took place in two waves: 102540 participants were enrolled in 1991-1997 (response rate 57\%), and 63232 participants in 2003-

872006 (response rate 48.4\%). The external validity of the NOWAC Study was found to be good (17).

88
Follow-up information has been collected up to two times after enrollment.

The NOWAC questionnaires targeted LNG-IUS use as from 1998 by the question: Have you ever used a hormone intrauterine device? A total of 145320 women completed a questionnaire during 1998-2006, either at enrollment or as part of follow-up. From these, we excluded 33182 that either did not answer the question on hormone intrauterine device or had a hysterectomy or oophorectomy; 4813 that either had prevalent cancer or died or emigrated before the start of follow-up; 2938 that indicated LNG-IUS use before the device was available in Norway, and seven for technical reasons. Thus the final study cohort consisted of 104380 women, of which 9146 were ever users of LNG-IUS.

\section{Exposure assessment}

In addition to questions on LNG-IUS (ever use, duration of use, age at first use, current use), we identified eight exposure variables associated with ovarian, endometrial, or breast cancer (18), regardless of their association with LNG-IUS use: age at start of follow-up (41-76 years, in 4-year 
100

101

102

103

104

105

106

107

108

109

110

111

112

113

114

115

116

117

increments), body mass index at enrollment (BMI, $<25 \mathrm{~kg} / \mathrm{m2}, \geq 25 \mathrm{~kg} / \mathrm{m2}$ ), physical activity level at enrollment (very low, low, intermediate, high, very high), maternal history of breast cancer (yes, no), age at menarche $(<12,12-14, \geq 15)$, ever use of OCs (yes, no), parity $(0,1-2,3-4, \geq 5)$, and menopausal status at start of follow-up (pre, peri, post, unknown). Unknown menopausal status was given to those who used hormone replacement therapy, those who indicated that menses had stopped due to "medication, illness, exercise, or other" and to those who did not answer the question.

\section{Outcomes}

Primary cancers were identified through linkage to the Cancer Registry of Norway using the International Classification of Diseases, Revision 7 (ICD-7) codes. All citizens were identified by their personal identification number upon contact with health care providers, who are obliged to report all cancer cases to the Cancer Registry of Norway. Cases were defined as cancer of the ovary including the fallopian tube (ICD-7 code 175), cancer of the uterine corpus (ICD-7 code 172), and cancer of the breast (ICD-7 code 170). In order to restrict the analyses to epithelial ovarian cancer and endometrial cancer, non-carcinoma cancers of the ovary and uterine corpus were excluded from the analyses $(n=62)$. Deaths and emigrations were identified through the Cause of Death Registry and Statistics Norway. Follow-up ended on 31 December 2015.

\section{Statistical analysis}

We calculated person-years (PY) of follow-up from the date of entrance into, until the date of exit from the study. Exit date was defined as the date of cancer diagnosis, emigration from Norway, death, or end of follow-up, whichever occurred first. We used chi-squared tests of independence to compare the characteristics of ever users and never users of LNG-IUS, and to compare selected characteristics of responders and non-responders of the question on LNG-IUS use.

We calculated crude cancer incidence rates with 95\% confidence intervals ( $\mathrm{Cls}$ ) assuming a Poisson distribution. Relative risks (RRs) and their $95 \%$ Cls were estimated with Poisson regression using a robust 
124 error estimate. Adjusted RR models were built in a stepwise backward manner by removing

125 nonsignificant covariates from the full model, with listwise deletion of participants with missing

126 information. Model fit was assessed by testing the deviance versus its assumed chi-squared distribution.

127 Statistical significance was defined as a test resulting in a $p$-value $<0.05$. We performed an additional

128 analysis of the association between LNG-IUS use and endometrial cancer, stratified by ever OC use (yes,

129 no), and did a Wald test of heterogeneity between the resulting RRs. We performed two additional

130 analyses of the association between LNG-IUS use and breast cancer: stratified by duration of use ( $\leq 5$ and

$131>5$ years), and stratified into current and former users at the start of follow-up.

132 The analyses were performed in SAS software version 9.4 (SAS Institute, Inc., Cary, NC, USA ).

133 Ethics

134 The Regional Ethics Committee, REK Nord, approved the NOWAC Study. Written information was

135 provided to the participants, and return of a completed questionnaire was considered as consent to

136 participate. Data storage is in compliance with the rules of the Norwegian Data Inspectorate. 


\section{RESULTS}

139

140

141

142

143

144

145

146

147

148

149

150

151

152

Median age at inclusion was 52 years. Mean follow-up time was 12.5 (standard deviation [SD] 3.7) years for a total of 1305435 PY. Among all ovarian and uterine corpus cancers, respectively $4 \%$ and $5 \%$ were non-carcinoma cancers and were excluded. Of the women in the study cohort, 9144 (9\%) reported LNGIUS use during or prior to the data collection period (1998-2007). Among ever users of the LNG-IUS, $85 \%$ reported the duration of use. Median age at starting LNG-IUS use was 44 years, and median duration was 4 years, with 59\% having used LNG-IUS for between 2 and 6 years. Compared to never users, ever users of LNG-IUS were younger at start of follow-up (Table 1).

The percentage of women that reported high or very high physical activity level was slightly higher among ever users of LNG-IUS (38\% versus $30 \%$ of never users) (Table 1). Ever use of OCs was more common among ever users of LNG-IUS (71\%) than never users (55\%), and nulliparity was more common among never users of LNG-IUS ( $10 \%$ vs. $3 \%$ among ever users). Menopausal status at start of follow-up was significantly different between the groups of LNG-IUS use, with $60 \%$ of never users reporting that they were postmenopausal, compared to $33 \%$ of ever users. Thirty percent $(n=2753)$ of ever users had unknown menopausal status, and of these, $85 \%$ were using LNG-IUS at the start of follow-up.

$$
\text { Non-responders to the LNG-IUS question ( } n=15442 \text { ) differed significantly from the study cohort on }
$$

all variables checked. Most notably they had a lower proportion of nullipara (Supplementary table S1).

\section{Levonorgestrel-releasing intrauterine system and cancer incidence}

Table 2 displays cancer incidences and risk estimates. The crude incidence rate of ovarian cancer among never users of LNG-IUS was 38.1 (95\% Cl: 34.7, 41.8). The crude incidence rate of ovarian cancer among ever users of LNG-IUS was 16.7 per 100000 PY (95\% Cl: 9.9, 26.4), with an age-adjusted RR of 0.49 (95\% $\mathrm{Cl}: 0.30,0.82)$ for ever versus never users. The final model for ovarian cancer included three significant covariates: age at start of follow-up, ever use of OCs, and menopausal status at start of follow-up. Parity 
was not significant in the model building, but qualified as a confounder and was included in the model. Adjustment for these covariates hardly changed the risk estimates (multivariable-adjusted RR 0.53 (95\% $\mathrm{Cl}: 0.32,0.88))$.

The reported duration of LNG-IUS use varied from less than 1 year to 14 years, with the latter value corresponding to the time difference between the introduction of the LNG-IUS in 1994 in Norway and the date of the last questionnaire (2008). There were 18 cases of ovarian cancer among ever users of LNG-IUS; 14 of these cases occurred in women who had been using LNG-IUS for less than 7 years, and 3 in women who did not report duration of use. Due to the low number of cases, duration-response estimates were not calculated.

The largest risk reduction was observed for endometrial cancer, with a multivariable RR of 0.22 (95\% Cl: $0.13,0.40)$ among ever users compared to never users of LNG-IUS. The final model for endometrial cancer adjusted for age at start of follow-up, BMI, physical activity level, OC use, parity, and menopausal status at start of follow-up. The stratified analysis showed that among ever users of OCs, ever users of LNG-IUS had a RR of endometrial cancer of $0.34(95 \% \mathrm{Cl}: 0.18,0.65)$ compared to never users of LNG-IUS. Among never users of OC, ever users of LNG-IUS had a RR of 0.08 ( $95 \% \mathrm{Cl}: 0.02,0.34$ compared to never users of LNG-IUS. However, these estimates were not significantly different $\left(p_{\text {heterogeneity }}=0.18\right)$.

For breast cancer, both the age-adjusted and the final adjusted model, which included age at start of follow-up, BMI, physical activity level, maternal history of breast cancer, OC use, and menopausal status at start of follow-up, showed no association with LNG-IUS use. The incidence rate of breast cancer was 275.7 per 100000 PY among ever users of LNG-IUS and 281.6 per 100000 PY among never users. The multivariable-adjusted RR of breast cancer among ever users of LNG-IUS was 1.03 (95\% Cl: 0.91, 1.17). Compared to never users, current users of LNG-IUS had a multivariable RR of breast cancer of 0.97 (95\% Cl: $0.80,1.19)$ and former users a RR of $0.79(95 \% \mathrm{Cl}: 0.64,0.98)$. Stratified by duration, ever users 
186 with up to 5 years of use had a multivariable RR of 1.06 ( $95 \% \mathrm{Cl}: 0.91,1.24)$ compared to never users.

187 Those with more than 5 years of use had a RR of 0.88 (95\% Cl: $0.68,1.16)$. Among ever users of LNG-IUS 188 with breast cancer, mean time since LNG-IUS cessation was 7.5 (SD 4.4) years ( $n=237$ ). For ever users of 189 LNG-IUS not diagnosed with cancer, mean time since cessation of use was 12.5 (SD 3.3) years.

190 When all cancers were added together to produce an estimate of the total effect of LNG-IUS use, in 191 ever users the RR of any hormone-related cancer was 0.86 ( $95 \% \mathrm{Cl}: 0.77,0.97)$ compared to never users. 192 193 


\section{DISCUSSION}

195 In this population-based prospective cohort study, women who reported ever use of LNG-IUS showed a

196 strongly reduced risk of both ovarian and endometrial cancer compared to those who did not. LNG-IUS

197 use was not associated with an increased risk of breast cancer.

198

Levonorgestrel and risk of ovarian cancer

Several studies have investigated the association between the use of intrauterine devices and

ovarian cancer, but most did not include LNG-IUS users, save one American, population-based, case-

202

control study, which consisted of 104 cases and 299 controls. This study included 14 LNG-IUS users, and

found a negative association between ever use of intrauterine device and ovarian cancer. When

analyzed by duration, only 4 or fewer years of use was protective (19). A Chinese prospective cohort

study that may have included LNG-IUS users found no association (20).

Two prospective cohort studies by Soini et al. described the association between LNG-IUS use and

ovarian cancer $(12,13)$. The most recent study $(12)$ was based on 77 ovarian cancer cases occurring in a

208 cohort of 93843 women who had been prescribed LNG-IUS for menorrhagia. The study did not adjust

209 for risk factors, and reported that the age-adjusted SIR of ovarian cancer among women with up to 5

210 years of LNG-IUS use was $0.59(95 \% \mathrm{Cl}: 0.47,0.73)$. Longer duration of use did not decrease the risk

211 much further. When the entire follow-up period was taken into account, the SIR was 0.49 (95\% Cl: 0.24,

$2120.87)$ for mucinous, $0.55(95 \% \mathrm{Cl}: 0.28,0.98)$ for endometrioid, and $0.75(95 \% \mathrm{Cl}: 0.55,0.99)$ for serous

213 ovarian carcinoma. After adjusting for important risk factors, our findings confirm those of Soini et al.,

214 and although our sample size did not permit analyses on histological subtypes, our adjusted results

215 strengthen the evidence of a causal association between LNG-IUS and decreased risk of ovarian cancer.

216 It is generally assumed that combined OCs prevent ovarian cancer by inhibiting ovulation (21) and

217 possibly by reducing menstrual bleeding (22). Sparse menstruations lead to less retrograde 
menstruation, which, by implanting as endometriosis, is thought to be a source of either endometrioid

219 carcinoma, clear cell carcinoma, or possibly low-grade serous carcinoma (23). By other mechanisms,

220 retrograde menstruation and follicular fluid released during ovulation may induce serous tubal

221 intraepithelial carcinoma (22), which potentially could enter the ruptured ovarian epithelium and,

222 stimulated by the hormone-rich milieu of the ovary, cause high-grade serous carcinoma (24).

223 Levonorgestrel is a potent progestin. LNG-IUS used in Norway at the time questionnaires were

224 completed initially release $20 \mu \mathrm{g}$ LNG per day, decreasing to $11 \mu \mathrm{g} /$ day for an average of $14 \mu \mathrm{g} /$ day over

225 a five-year period (25). LNG-IUS exerts its contraceptive effect by suppressing the endometrium,

226 thickening the cervical mucus, and, partly, by inhibiting ovulation through the hypothalamic-pituitary

227 axis (26). Most LNG-IUS users have light menstruations and 20-50\% become amenorrheic (27). In the

228 present study, 30\% of LNG-IUS users had unknown menopausal status, compared to $5 \%$ of non-users. In

229 an ultrasound study of 22 women, of which one-third were amenorrheic after 7 or more years of LNG-

230 IUS use, approximately $30 \%$ of amenorrheic women and $60 \%$ of still menstruating women had ovulatory

231 cycles with follicular rupture (26).

232 Risch (28) argued that, since the protective effect of progestin-only contraceptives, which do not

233 completely suppress ovulation, is comparable to the effect of combined OCs on ovarian cancer,

234 progestogens likely have a direct anti-tumorigenic effect on ovarian cancer. Such a concept was

235 supported by Merritt et al. (29) notably with regard to high natural progesterone levels during

236 pregnancy, though the effects of natural progesterone and those of synthetic progestins are not

237 superimposable. The LNG-IUS alleviates symptoms of endometriosis, and Lockhat et al. (30) showed

238 that in addition to the vascular delivery of levonorgestrel to endometriotic implants, direct contact with

239 levonorgestrel in peritoneal fluid (transferred to this fluid via blood, not by diffusion from the uterine

240 cavity) likely plays a significant role. A similar direct effect on ovarian tumors or tumor precursor cells is

241 also possible (31). This hypothesis, however, does not correspond with a Danish population-based case- 
242 control study (21) nor with a previous study from the NOWAC cohort (32), both of which found that only

243 use of combined OCs, not oral progestogens alone, prevents ovarian cancer. Faber et al. (21) concluded

244 that OCs prevent ovarian cancer through the inhibition of ovulation. It is plausible that the preventive

245 effect of LNG-IUS on ovarian cancer works through partial inhibition of both ovulation and

246 menstruation.

247

248

Levonorgestrel and risk of endometrial cancer

249 Our adjusted results also confirm the observations of Soini et al. (13) for endometrial cancer. That

250 study adjusted for smoking, diet and alcohol consumption, socioeconomic status, and physical activity,

251 and reported a SIR of endometrial cancer of $0.46(95 \% \mathrm{Cl}: 0.33,0.64)$ in LNG-IUS users compared to the

252 general population. In a pooled analysis of four cohort and 14 case-control studies, Felix et al. (33)

253 calculated the association between use of different intrauterine devices and the risk of endometrial

254 cancer and found no association with LNG-IUS. However, due to the low number of women in the LNG-

255 IUS exposure group, they disregarded this result and called for further studies.

256

257 The anti-proliferative effect of LNG-IUS is superior to that of oral progestins in the treatment of

258 endometrial hyperplasia (15), and a protective effect of this device on endometrial cancer in the general

259 population is to be expected. Our results indicate the size of the risk reduction in a cohort

260 representative of the general population. Since the proportion of ever users of OCs was significantly

261 different among ever and never users of LNG-IUS, we performed an analysis stratified by ever OC use.

262 The difference was non-significant, but suggestive of a stronger protective effect of LNG-IUS among

263 never users of OCs. 
266 Contrary to Soini et al. (14), we did not observe an increased risk of breast cancer among LNG-IUS

267 users. Soini et al. (14) reported a clear increased risk of certain types of breast cancer, but did not

268 present SIRs of total breast cancer. In the earlier study by Soini et al. (13), the SIR of total breast cancer

269 was $1.19(95 \% \mathrm{Cl}: 1.13,1.25)$. In all three studies by Soini et al. (12-14) follow-up ended at age 55 years.

270 The discrepancy between our findings and those of Soini et al. (14) could be due to their lack of

271 adjustment, although adjustment had little effect on our estimates.

272 In a recent nested case-control study of women in the Norwegian breast cancer screening program

273 (aged 50-69 years), Ellingjord-Dale et al. (34) did not find an association between duration of IUD use

274 and overall risk of breast cancer by duration of use (in intervals), although there was a statistically

275 significant trend. The results indicated increased and decreased risks of different breast cancer

276 subtypes. This study did not differentiate between types of intrauterine devices, but assuming a

277 population-representative sample and data collected from 2006 onwards, LNG-IUS users constituted a

278 large fraction of intrauterine device users (11). When we stratified on duration of use (up to 5 and more

279 than 5 years), we observed no association with breast cancer in either stratum. We did not study breast

280 cancer subtypes, and we did not test for trend.

281

A recent prospective cohort study showed that current and recent users of LNG-IUS had an

282 increased risk of breast cancer compared to never users of hormonal contraceptives (RR 1.21; 95\% Cl:

283 1.11, 1.33). This study included all women aged 15-49 in Denmark, and adjusted for age, calendar year,

284 education, polycystic ovary syndrome, endometriosis, parity, and family history of premenopausal

285 cancer of the breast or ovary. Our null finding remained when we restricted the analyses to current

286 users of LNG-IUS. However, in our study, few participants were younger than 46 years. Moreover, the

287 mean duration of LNG-IUS use was 4 years, and average time since cessation of use was 7.5 years. When

$288 M \emptyset r c h$ et al stratified by duration of use and time since cessation, women in the corresponding category 
did not have increased and risk of breast cancer. Mørch et al. found that more than 5 years of use was

290 associated with increased cancer risk, and the risk lasted up to 10 years after cessation of use (9). In our analysis stratified on duration we could not reproduce this finding.

Among previous studies, a Finnish case-control study of 9537 breast cancer cases and 21598 controls adjusted for age at menarche, smoking, alcohol use, BMI, and family history of breast cancer and found a positive association between ever use of LNG-IUS and breast cancer in postmenopausal women (aged 51-64), while for premenopausal women no association was observed (35). The authors mentioned the possible presence of selection bias, as some practitioners, at least in Finland (this is also the case in Norway), have regarded the LNG-IUS as a preferable option for women with an increased risk of breast cancer.

Strengths and limitations

301 Strengths of this study include its prospective design, inclusion of lifestyle information, and a population 302 based study cohort with women who were likely using the LNG-IUS for both contraceptive and medical 303 reasons. BMI and OCs were validated by test-retest in a subset of participants (Skeie 2015, Lund, 304 Dumeaux et al. 2008), and physical activity and menopausal status by measurements (Borch 305 2012,Waaseth 2008). Number of children was validated by Lund, Kumle (17). The LNG-IUS variable was 306 not validated, nor was maternal history of breast cancer and age at menarche. Compared to non-

307 responders, responders were at a disadvantage with regard to some risk factors for the cancers in this 308 study (lower age at menarche and nulliparity), but also had favorable characteristics (proportion of ever 309 OC users and maternal history of breast cancer). We included OC use as a dichotomous variable, as 310 analyzing OC use by duration did not change the estimate of the main exposure. We did not adjust for 311 time since OC use. Insufficient adjustment for this, and for use of other hormonal contraceptives, may 312 have caused residual confounding in our estimates. 
The use of cancer registry data ensured near complete follow-up of cancer cases. However, due to

314 the strong protective effect of LNG-IUS, the study had a limited number of ovarian and endometrial

315 cancer cases. We were not able to calculate specific rates by subtype, nor could we analyze duration

316 effects on these cancer types.

317 The mean age at enrollment was lower among ever users of LNG-IUS than never users. The

318 gynecological practice of removing or leaving LNG-IUS in place at the time of menopause, varies;

319 nevertheless, even if left in place, its protective effect, if any, could be transitory, potentially delaying

320 the "natural" appearance of ovarian cancer. We created a Lexis diagram of the distribution of ovarian

321 cancer incidence in both ever users and never users of LNG-IUS, which showed a lower, but parallel

322 incidence rate among all LNG-IUS users aged less than 65 years, and a decreased incidence rate among

323 those aged 65 years or over, as compared to never users. However, among ever users of LNG-IUS, there

324 was one case that occurred after age 64 years, which introduces uncertainty into the estimation.

325 This is one of the few epidemiological studies that presents data specifically on LNG-IUS use, with

326 estimates generalizable to the general female population of Norway. However, we used self-reported

327 exposure data, which introduces a risk of misclassification. Considering the prescription routines, it is

328 likely that women were counselled by their physician and required to make a choice, and thus were

329 aware of which type of intrauterine device they were using. Nevertheless, we excluded women who

330 indicated using LNG-IUS before it was on the market.

\section{CONCLUSION}

332 This study shows that a relatively short period of LNG-IUS use is associated with an almost halved risk of

333 ovarian cancer, while the risk of breast cancer remains unchanged. Our results are in agreement with

334 existing data, and show a negative association in a cohort of women where the majority was older than

335 in previous studies. Although these findings suggest that LNG-IUS should be considered for inclusion in 
the ovarian cancer prevention strategy for normal-risk women in addition to OCs (36), an updated meta-

337 analysis of the effect of LNG-IUS on breast cancer is needed before firm conclusions can be drawn.

340 Author contributions

341 EL and HMB conceived the study. MJ contributed to designing the analyses, interpreted results and

342 drafted the paper. EL and JCT oversaw the analyses, interpreted results and critically revised the paper.

343 TB designed the analyses, carried out analyses, and interpreted the results. HMB carried out preliminary

344 analyses, and MAA carried out final analyses. EL is the PI of the NOWAC Study. All authors read and

345 approved the final manuscript.

346 Acknowledgements

347 While employed at UiT, Nicolle Mode contributed to the design of, and scripts for, statistical analysis, as 348 well as contributing text to this paper. The authors are supported by the Faculty of Health Sciences, UiT

349 The Arctic University of Norway, Troms $\varnothing$, Norway. JCT has a full-time position at the Medical Faculty,

350 Paris Descartes University, and is the beneficiary of a part-time position at UiT. The funding bodies had 351 no role in the design, collection, analysis, or interpretation of data; in the writing of the manuscript, or 352 the decision to submit the manuscript for publication. We sincerely thank the women who participate in 353 the NOWAC Study.

354 Conflict of interest statement

355 We declare that we have no conflicting interests. 


\section{REFERENCES}

359 1. Zou G. A Modified Poisson Regression Approach to Prospective Studies with Binary Data. Am J Epidemiol. 2004;159(7):702-6.

361 2. Ferlay J SI, Ervik M, Dikshit R, Eser S, Mathers C, Rebelo M, Parkin DM, Forman D, Bray, F.

362 GLOBOCAN 2012 v1.0, Cancer Incidence and Mortality Worldwide: IARC CancerBase No. 11. Lyon,

363 France: International Agency for Research on Cancer (IARC); 2013. Contract No.: 08-20.

$3643 . \quad$ SEER. Cancer Stat Facts: Ovarian Cancer: National Cancer Institute - The Surveillance,

365 Epidemiology, and End Results (SEER) Program 2017 [Available

366 from: https://seer.cancer.gov/statfacts/html/ovary.html.

3674 4. Cancer Registry of Norway. Cancer in Norway 2015 - Cancer incidence, mortality, survival and

368 prevalence in Norway. Oslo: Cancer Registry of Norway; 2016.

$3695 . \quad$ Long Roche KC, Abu-Rustum NR, Nourmoussavi M, Zivanovic O. Risk-reducing salpingectomy: Let

370 us be opportunistic. Cancer. 2017;123(10):1714-20.

371 6. Beral V, Doll R, Hermon C, Peto R, Reeves G. Ovarian cancer and oral contraceptives:

372 collaborative reanalysis of data from 45 epidemiological studies including 23,257 women with ovarian

373 cancer and 87,303 controls. Lancet. 2008;371(9609):303-14.

$3747 . \quad$ Dossus L, Allen N, Kaaks R, Bakken K, Lund E, Tjonneland A, et al. Reproductive risk factors and

375 endometrial cancer: the European Prospective Investigation into Cancer and Nutrition. Int J Cancer.

376 2010;127(2):442-51.

377 8. Collaborative Group on Hormonal Factors in Breast Cancer. Breast cancer and hormonal

378 contraceptives: collaborative reanalysis of individual data on 53297 women with breast cancer and 100

379239 women without breast cancer from 54 epidemiological studies. The Lancet. 1996;347(9017):1713-

38027.

3819 9. Mørch LS, Skovlund CW, Hannaford PC, Iversen L, Fielding S, Lidegaard $\varnothing$. Contemporary

382 Hormonal Contraception and the Risk of Breast Cancer. N Engl J Med. 2017;377(23):2228-39.

383 10. Havrilesky LJ GJ, Moorman PG, Coeytaux RR, Peragallo Urrutia R, Lowery WJ, Dinan M, McBroom

384 AJ, Wing L, Musty MD, Lallinger KR, Hasselblad V, Sanders GD, Myers ER. Oral Contraceptive Use for the

385 Primary Prevention of Ovarian Cancer. Rockville, MD: Agency for Healthcare Research and Quality.: Duke Evidence-based Practice Center; 2013 June. Report No.: Evidence Report/Technology Assessment No. 212. Contract No.: AHRQ Publication No. 13-E002-EF.

11. Lindh I, Skjeldestad FE, Gemzell-Danielsson K, Heikinheimo O, Hognert H, Milsom I, et al. Contraceptive use in the Nordic countries. Acta Obstet Gynecol Scand. 2017;96(1):19-28.

12. Soini T, Hurskainen R, Grenman S, Maenpaa J, Paavonen J, Pukkala E. Impact of levonorgestrelreleasing intrauterine system use on the cancer risk of the ovary and fallopian tube. Acta Oncol. 2016:14.

13. Soini T, Hurskainen R, Grenman S, Maenpaa J, Paavonen J, Pukkala E. Cancer risk in women using the levonorgestrel-releasing intrauterine system in Finland. Obstet Gynecol. 2014;124(2 Pt 1):2929.

14. Soini T, Hurskainen R, Grénman S, Mäenpää J, Paavonen J, Joensuu H, et al. Levonorgestrelreleasing intrauterine system and the risk of breast cancer: A nationwide cohort study. Acta Oncol. 398 2016;55(2):188-92.

399 15. Orbo A, Vereide A, Arnes M, Pettersen I, Straume B. Levonorgestrel-impregnated intrauterine

400

401

402

403 device as treatment for endometrial hyperplasia: a national multicentre randomised trial. BJOG. 2014;121(4):477-86.

16. Lund E, Dumeaux V, Braaten T, Hjartaker A, Engeset D, Skeie G, et al. Cohort profile: The Norwegian Women and Cancer Study--NOWAC--Kvinner og kreft. Int J Epidemiol. 2008;37(1):36-41. 
17. Lund E, Kumle M, Braaten T, Hjartaker A, Bakken K, Eggen E, et al. External validity in a population-based national prospective study--the Norwegian Women and Cancer Study (NOWAC). Cancer Causes Control. 2003;14(10):1001-8.

18. Stewart B, Wild C, editors. World Cancer Report 2014. Lyon, France: International Agency for Research on Cancer; 2014.

19. Ness RB, Dodge RC, Edwards RP, Baker JA, Moysich KB. Contraception Methods, Beyond Oral Contraceptives and Tubal Ligation, and Risk of Ovarian Cancer. Ann Epidemiol. 2011;21(3):188-96.

20. Dorjgochoo T, Shu XO, Li HL, Qian HZ, Yang G, Cai H, et al. Use of oral contraceptives, intrauterine devices and tubal sterilization and cancer risk in a large prospective study, from 1996 to 2006. Int J Cancer. 2009;124(10):2442-9.

21. Faber MT, Jensen A, Frederiksen K, Glud E, Hogdall E, Hogdall C, et al. Oral contraceptive use and impact of cumulative intake of estrogen and progestin on risk of ovarian cancer. Cancer Causes Control. 2013;24(12):2197-206.

22. Vercellini $P$, Crosignani $P$, Somigliana E, Vigano P, Buggio L, Bolis G, et al. The 'incessant menstruation' hypothesis: a mechanistic ovarian cancer model with implications for prevention. Hum Reprod. 2011;26(9):2262-73.

23. Pearce CL, Templeman C, Rossing MA, Lee A, Near AM, Webb PM, et al. Association between endometriosis and risk of histological subtypes of ovarian cancer: a pooled analysis of case-control studies. The Lancet Oncology. 2012;13(4):385-94.

24. Kurman RJ, Shih le M. The origin and pathogenesis of epithelial ovarian cancer: a proposed unifying theory. Am J Surg Pathol. 2010;34(3):433-43.

25. ESHRE Capri Workshop Group. Intrauterine devices and intrauterine systems. Hum Reprod Update. 2008;14(3):197-208.

26. Barbosa I, Olsson SE, Odlind V, Goncalves T, Coutinho E. Ovarian function after seven years' use of a levonorgestrel IUD. Adv Contracept. 1995;11(2):85-95.

27. Hidalgo M, Bahamondes L, Perrotti M, Diaz J, Dantas-Monteiro C, Petta C. Bleeding patterns and clinical performance of the levonorgestrel-releasing intrauterine system (Mirena) up to two years1 1Mirena is a registered trademark of Leiras Oy, Turku, Finland. Contraception. 2002;65(2):129-32. 28. Risch HA. Hormonal Etiology of Epithelial Ovarian Cancer, With a Hypothesis Concerning the Role of Androgens and Progesterone. JNCI: Journal of the National Cancer Institute. 1998;90(23):177486.

29. Merritt MA, De Pari M, Vitonis AF, Titus LJ, Cramer DW, Terry KL. Reproductive characteristics in relation to ovarian cancer risk by histologic pathways. Hum Reprod. 2013;28(5):1406-17.

30. Lockhat FB, Emembolu JE, Konje JC. Serum and peritoneal fluid levels of levonorgestrel in women with endometriosis who were treated with an intrauterine contraceptive device containing levonorgestrel. Fertil Steril. 2005;83(2):398-404.

31. Rodriguez GC, Nagarsheth NP, Lee KL, Bentley RC, Walmer DK, Cline M, et al. Progestin-induced apoptosis in the Macaque ovarian epithelium: differential regulation of transforming growth factor-beta. J Natl Cancer Inst. 2002;94(1):50-60.

32. Kumle M, Weiderpass E, Braaten T, Adami HO, Lund E. Risk for invasive and borderline epithelial ovarian neoplasias following use of hormonal contraceptives: the Norwegian-Swedish Women's Lifestyle and Health Cohort Study. Br J Cancer. 2004;90(7):1386-91.

33. Felix AS, Gaudet MM, Vecchia CL, Nagle CM, Shu XO, Weiderpass E, et al. Intrauterine devices and endometrial cancer risk: A pooled analysis of the Epidemiology of Endometrial Cancer Consortium. Int J Cancer. 2014.

34. Ellingjord-Dale M, Vos L, Tretli S, Hofvind S, dos-Santos-Silva I, Ursin G. Parity, hormones and breast cancer subtypes - results from a large nested case-control study in a national screening program. Breast Cancer Res. 2017;19(1):10. 
$452 \quad 35 . \quad H e i k k i n e n$ S, Koskenvuo M, Malila N, Sarkeala T, Pukkala E, Pitkäniemi J. Use of exogenous 453 hormones and the risk of breast cancer: results from self-reported survey data with validity assessment. 454 Cancer Causes Control. 2016;27(2):249-58.

455 36. Walker JL, Powell CB, Chen L-m, Carter J, Bae Jump VL, Parker LP, et al. Society of Gynecologic 456 Oncology recommendations for the prevention of ovarian cancer. Cancer. 2015;121(13):2108-20.

457

458

459

460 
Table 1 Characteristics of ever users ( $\mathrm{N}=9144)$ and never users ( $\mathrm{N}=95$ 174) of the levonorgestrelreleasing intrauterine system (LNG-IUS) in the Norwegian Women and Cancer Study, 1998-2015

\begin{tabular}{|c|c|c|c|c|c|c|}
\hline \multirow[t]{2}{*}{ Characteristics } & & \multicolumn{4}{|c|}{ LNG-IUS } & \multirow[b]{2}{*}{ p-value } \\
\hline & & \multicolumn{2}{|c|}{ Ever users } & \multicolumn{2}{|c|}{ Never users } & \\
\hline \multirow{7}{*}{$\begin{array}{l}\text { Age at start of follow-up } \\
\text { (years) }\end{array}$} & $41-45$ & 1271 & $14 \%$ & 11177 & $12 \%$ & $<0.01$ \\
\hline & $46-50$ & 3855 & $42 \%$ & 21581 & $23 \%$ & \\
\hline & $51-55$ & 3051 & $33 \%$ & 30526 & $32 \%$ & \\
\hline & $56-60$ & 795 & $9 \%$ & 18589 & $20 \%$ & \\
\hline & $61-65$ & 145 & $2 \%$ & 7811 & $8 \%$ & \\
\hline & $66-70$ & 20 & $<1 \%$ & 3012 & $3 \%$ & \\
\hline & $71-76$ & 7 & $<1 \%$ & 2478 & $3 \%$ & \\
\hline \multirow[t]{3}{*}{ Body mass index $\left(\mathbf{k g} / \mathbf{m}^{2}\right)$} & $<25$ & 5295 & $58 \%$ & 54133 & $57 \%$ & 0.18 \\
\hline & $\geq 25$ & 3637 & $40 \%$ & 38306 & $40 \%$ & \\
\hline & missing & 212 & & 2735 & & \\
\hline \multirow[t]{6}{*}{ Physical activity level } & very low & 248 & $3 \%$ & 3506 & $4 \%$ & $<0.01$ \\
\hline & Low & 1518 & $17 \%$ & 18200 & $19 \%$ & \\
\hline & intermediate & 3479 & $38 \%$ & 36971 & $39 \%$ & \\
\hline & High & 2972 & $33 \%$ & 23871 & $25 \%$ & \\
\hline & very high & 556 & $6 \%$ & 4796 & $5 \%$ & \\
\hline & missing & 371 & & 7830 & & \\
\hline $\begin{array}{l}\text { Maternal history of breast } \\
\text { cancer }\end{array}$ & Yes & 478 & $5 \%$ & 5032 & $5 \%$ & 0.80 \\
\hline \multirow[t]{4}{*}{ Age at menarche (years) } & $<12$ & 811 & $9 \%$ & 8428 & $9 \%$ & 0.01 \\
\hline & $12-14$ & 6646 & $73 \%$ & 67897 & $71 \%$ & \\
\hline & $\geq 15$ & 1543 & $17 \%$ & 17364 & $18 \%$ & \\
\hline & missing & 144 & & 1485 & & \\
\hline $\begin{array}{l}\text { Ever use of oral } \\
\text { contraceptives }\end{array}$ & Yes & 6476 & $71 \%$ & 52259 & $55 \%$ & $<0.01$ \\
\hline \multirow[t]{4}{*}{ Parity } & None & 307 & $3 \%$ & 9231 & $10 \%$ & $<0.01$ \\
\hline & $1-2$ & 5502 & $60 \%$ & 49935 & $52 \%$ & \\
\hline & $3-4$ & 3173 & $35 \%$ & 32762 & $34 \%$ & \\
\hline & $\geq 5$ & 162 & $2 \%$ & 3246 & $3 \%$ & \\
\hline \multirow[t]{4}{*}{$\begin{array}{l}\text { Menopausal status at } \\
\text { enrollment }\end{array}$} & Pre & 2125 & $23 \%$ & 24323 & $26 \%$ & $<0.01$ \\
\hline & Peri & 1206 & $13 \%$ & 8533 & $9 \%$ & \\
\hline & Post & 3060 & $33 \%$ & 57128 & $60 \%$ & \\
\hline & Unknown & 2753 & $30 \%$ & 5190 & $5 \%$ & \\
\hline
\end{tabular}

* P-value from a chi-square test of independence, excluding missing value 
Table 2 Site-specific cancer incidence rates and relative risks comparing ever users (person-years [PY] =107 701) and never users (PY=1 197 734) of the levonorgestrel-releasing intrauterine system (LNG-IUS) in the Norwegian Women and Cancer Study

\begin{tabular}{|c|c|c|c|c|c|}
\hline Cancer type & $\begin{array}{c}\text { LNG-IUS user } \\
\text { status }\end{array}$ & Cancer cases & $\begin{array}{r}\text { Incidence rate per } \\
100000 \text { PY (95\% CI) }\end{array}$ & $\begin{array}{r}\text { Age-adjusted RR } \\
(95 \% \mathrm{CI})\end{array}$ & $\begin{array}{r}\text { Multivariable-adjusted } \\
\text { RR (95\% CI) }\end{array}$ \\
\hline \multirow[t]{2}{*}{ Epithelial ovarian } & Ever & 18 & $16.7(9.9,26.4)$ & $0.49(0.30,0.82)$ & $0.53(0.32,0.88)^{\mathrm{a}}$ \\
\hline & Never & 457 & $38.1(34.7,41.8)$ & & \\
\hline \multirow[t]{2}{*}{ Endometrial } & Ever & 15 & $13.9(7.8,23.0)$ & $0.19(0.11,0.40)$ & $0.22(0.13,0.40)^{\mathrm{b}}$ \\
\hline & Never & 839 & $70.0(65.4,74.9)$ & & \\
\hline \multirow[t]{2}{*}{ Breast } & Ever & 297 & $275.7(245.3,309.0)$ & $1.02(0.90,1.15)$ & $1.03(0.91,1.17)^{\mathrm{c}}$ \\
\hline & Never & 3373 & $281.6(272.2,291.3)$ & & \\
\hline Combined (ovarian, & Ever & 330 & $306.4(274.2,341.3)$ & $0.84(0.74,0.94)$ & $0.86(0.77,0.97)^{\mathrm{d}}$ \\
\hline breast, endometrial) & Never & 4669 & 389.7 (378.7, 401.2) & & \\
\hline
\end{tabular}


Supplementary table 1 Selected characteristics of responders and non-responders to the question 'Have you ever used a hormone intrauterine device (IUD)?' in the Norwegian Women and Cancer Study, 19982015

\begin{tabular}{|c|c|c|c|c|c|c|}
\hline \multirow[t]{2}{*}{ Characteristics } & \multirow[b]{3}{*}{ Yes } & \multicolumn{4}{|c|}{ Have you ever used a hormone IUD? } & \multirow[b]{2}{*}{ p-value } \\
\hline & & \multicolumn{2}{|c|}{$\begin{array}{c}\text { Responders } \\
104380\end{array}$} & \multicolumn{2}{|c|}{$\begin{array}{c}\text { Non-responders } \\
15442\end{array}$} & \\
\hline $\begin{array}{l}\text { Maternal history of breast } \\
\text { cancer }\end{array}$ & & 5515 & $5 \%$ & 940 & $6 \%$ & $<.001$ \\
\hline \multirow[t]{4}{*}{ Age at menarche (years) } & $<12$ & 9246 & $9 \%$ & 1318 & $8 \%$ & $<.001$ \\
\hline & $12-14$ & 74584 & $71 \%$ & 10767 & $70 \%$ & \\
\hline & $\geq 15$ & 18920 & $18 \%$ & 3030 & $20 \%$ & \\
\hline & missing & 1630 & $2 \%$ & 327 & $2 \%$ & \\
\hline $\begin{array}{l}\text { Ever use of oral } \\
\text { contraceptives }\end{array}$ & Yes & 58761 & $56 \%$ & 8366 & $54 \%$ & $<.001$ \\
\hline \multirow[t]{4}{*}{ Parity } & None & 9547 & $9 \%$ & 939 & $6 \%$ & $<.001$ \\
\hline & $1-2$ & 55466 & $53 \%$ & 8546 & $55 \%$ & \\
\hline & $3-4$ & 35957 & $35 \%$ & 5535 & $36 \%$ & \\
\hline & $\geq 5$ & 3410 & $3 \%$ & 422 & $3 \%$ & \\
\hline
\end{tabular}

* P-value from a chi-square test of independence, excluding missing value 